\title{
Application of sub-segmentation enhancement in pore detection in soil CT images
}

\section{Aplicación de la mejora de la sub-segmentación en la detección de poros en imágenes de CT de suelo}

\author{
ARREGUIN-JUÁREZ, Miguel $\dagger^{\prime}$, QUINTANILLA-DOMINGUEZ, Joel*’', OJEDA-MAGAÑA, \\ Benjamín” and TARQUIS-ALFONSO, Ana María"”
}

'Universidad Politécnica de Juventino Rosas, Master's in engineering in the Intelligent Systems area. Mexico.

"Universidad de Guadalajara, Project Engineering Department, CUCEI. Mexico.

"'Universidad Politécnica de Madrid, GSC \& CEIGRAM, ETSIAAB. Spain.

ID $1^{\text {st }}$ Author: Miguel, Arreguin-Juárez / ORC ID: 0000-0003-2312-2695

ID $1^{\text {st }}$ Co-author: Joel, Quintanilla-Domínguez / ORC ID: 0000-0003-2442-2032

ID $2^{\text {nd }}$ Co-author: Benjamín, Ojeda-Magaña / ORC ID: 0000-0001-9476-9993

ID $3^{\text {rd }}$ Co-author: Ana María, Tarquis-Alfonso / ORC ID: 0000-0003-2336-5371

DOI: $10.35429 /$ JTI.2021.22.8.9.19

Received January 10, 2021; Accepted June 30, 2021

\begin{abstract}
Computed Tomography imaging is a non-invasive alternative to observe soil structures, mainly the pore space. The porous space corresponds in the image of the soil to an empty or free space in the sense that there is no material present but only fluids and the transport of these depends on the porous spaces in the soil, for this reason it is important to identify the regions that correspond to the pore areas. Due to this, this article presents a methodology based on digital image processing techniques with the objective of segmenting porous spaces in soil images. The methodology consists mainly of two stages. The first is an image contrast enhancement through a nonlinear adaptive transformation function and the second is an image segmentation through a technique known as subsegmentation enhancement which is based on the Fuzzy Possibilist C clustering algorithm. -Medias (Possibilistic Fuzzy C-Means, PFCM). The results obtained in the segmentation stage are compared with the technique known as sub-segmentation or conventional sub-segmentation, which is also based on the PFCM hybrid algorithm. In this article it is shown that both segmentation techniques are robust, but nevertheless the area of opportunity of the classic sub-segmentation and the improvement process that results in the new sub-segmentation or improvement of the subsegmentation are also shown. segmentation.
\end{abstract}

Digital image processing, contrast enhancement, segmentation, clustering algorithms

\begin{abstract}
Resumen
La imagen por tomografía computarizada es una alternativa no invasiva para observar las estructuras del suelo, principalmente el espacio poroso. El espacio poroso corresponde en la imagen del suelo a un espacio vacío o libre en el sentido de que no hay material presente sino sólo fluidos y el transporte de éstos depende de los espacios porosos en el suelo, por esta razón es importante identificar las regiones que corresponden a las zonas de poros. Debido a esto, en este artículo se presenta una metodología basada en técnicas de procesamiento digital de imágenes con el objetivo de segmentar espacios porosos en imágenes de suelo. La metodología se compone principalmente de dos etapas. La primera es una mejora de contraste de imagen mediante una función de transformación adaptativa no lineal y la segunda en una segmentación de imagen por medio de una técnica conocida como mejora de la sub-segmentación la cual se basada en el algoritmo de agrupamiento Posibilista Difuso CMedias (Possibilistic Fuzzy C-Means, PFCM). Los resultados obtenidos en la etapa de segmentación son comparados con la técnica conocida como sub-segmentación o sub-segmentación convencional, la cual también está basada en el algoritmo hibrido PFCM. En el presente artículo se muestra que ambas técnicas de segmentación son robustas, sin embargo, también se muestran el área de oportunidad de la sub-segmentación clásica y el proceso de mejora que da como resultado la nueva sub-segmentación o mejora de la sub-segmentación.
\end{abstract}

Procesamiento digital de imágenes, mejora de contraste, segmentación, algoritmos de agrupamiento

Citation: ARREGUIN-JUÁREZ, Miguel, QUINTANILLA-DOMINGUEZ, Joel, OJEDA-MAGAÑA, Benjamín and TARQUIS-ALFONSO, Ana María. Application of sub-segmentation enhancement in pore detection in soil CT images. Journal of Technology and Innovation. 2021. 8-22:9-19.

\footnotetext{
* Correspondence of the Author (Email: jquintanilla_ptc@upjr.edu.mx)

$\dagger$ Researcher contributing as first author.
} 


\section{Introduction}

The study and analysis of soil samples is of utmost importance for the identification of the pore structure of a soil segment, this provides information on the mechanics of the soil and displays information of interest. Another importance of soil analysis is the study of exploring the influence of the soil structure, that is, the spatial arrangement of the soil pores.

Soil analysis focuses on performing different tasks, such as segmentation, classification, and interpretation. In some cases it is also necessary to carry out a preprocessing which improves or prepares the image so that it can be processed in such a way that for the segmentation and interpretation algorithms it facilitates the labeling of pixels and the processing of the data. According to (Vogel, 1996) segmentation implies the identification of objects in images. For the segmentation of images, a data grouping process is used which searches for pixels with similar intensities of gray in order to assign them into homogeneous groups. Subsequently, the similarity between pixels is evaluated according to a measure of distance between each pixel and a prototype that represents each object or region, according to the above, each pixel is assigned to the group with the closest prototype or that has something in particular, normally it is expects the distance between patterns in the same clustering to be significantly smaller than the distance between different clustering patterns. For the grouping process, we have a set of vectors $\left\{\mathrm{x}_{-} 1, \ldots, \mathrm{x} \_\mathrm{n}\right\}$, which represent the objects and from this we want to obtain the set of groups $\{1, \ldots, n\}$ that encompasses them. The problem is that a priori it is not known how the vectors are distributed in the classes, or even how many classes there will be, therefore, from the given set of vectors of characteristics, we try to group these vectors into groups, according to with the similarities that have been found (Moreiro, 2002). All groups have a centroid, which represents the objects of the group to which it belongs. The similarity to the objects in the cluster with respect to their centroid is measured by the similarity function, for example, the Euclidean distance. For the partition grouping the objective is to obtain a partition of the objects into groups in such a way that all the objects belong to one of the k possible groups and that on the other hand the groups are disjoint.
Partitional algorithms that focus on partitioning data are classified as: hard or strict, fuzzy, and possible. The main characteristic of a strict partition is that it is based on a Bayesian classification, that is, the algorithm strictly decides (according to certain properties and characteristics) whether or not the data of a certain analysis belongs to a certain data group. In accordance with the above, taking into account the logical values $[1,0]$, the following could be assumed: 1 the data belongs and 0 does not belong to a certain group (discrete data). In the case of fuzzy partitioning, a piece of data is capable of belonging to more than one group, with a fuzzy degree of membership between the values $[0,1]$ where the sum of the membership values is equal to 1 (Continuous data). Finally, in the possibilistic partition, its main characteristic is that it alleviates the restriction of the sum of the membership values in the fuzzy partition that is equal to 1 , in addition to that, in each data, it obtains a possible value or degree of membership to the which is also known as a typical value for each of the groups that is independent of the other groups, this algorithm can have a degree of membership of the data between the values $[0,>1]$.

For the segmentation of images by means of clustering algorithms, different techniques have been used that are based on the family of K-Means algorithms (MacQueen, 1967), which is one of the unsupervised strict partition learning algorithms that It is used to solve the data grouping problem, this means that the algorithm performs the grouping of the data based on a criterion of minimum distance between them and the center of the group. Another highly relevant algorithm for data grouping is the Fuzzy C-Means or Fuzzy CMeans (FCM) which was proposed by (Dunn, 1973). Later (Bezdek, 1981) generalized this algorithm and explained that the Boolean logic on which the K-Means algorithm is based is known as the most accurate and that despite its accuracy advantages, it has a disadvantage, which is not being able to reproduce the patterns of human thought, while the fuzzy logic on which the FCM algorithm is based is designed to react to continuous changes in the variable to be controlled and is not restricted to only two values of 0 and 1 This which indicates that it is a fuzzy or soft partition algorithm. An important characteristic of FCM is that its foundations are based on the theory of fuzzy sets that was proposed by (Zadeh, 1965).

ARREGUIN-JUÁREZ, Miguel, QUINTANILLADOMINGUEZ, Joel, OJEDA-MAGAÑA, Benjamín and TARQUIS-ALFONSO, Ana María. Application of subsegmentation enhancement in pore detection in soil CT images. Journal of Technology and Innovation. 2021 
To solve the proposal of this research, it is not pertinent to use the K-Means clustering algorithm, however, a hybrid algorithm that focuses on the characteristics of the FCM and PCM algorithms will be used, which is called Fuzzy Posibilist C-Means. (Possibilistic Fuzzy C-Means, PFCM) (Pal, 2005) which verify that the algorithm is robust through a set of standard data. After the execution of the algorithm, a method is used which is called subsegmentation, it is based on the concept of typical values or typicality, that is, it allows finding the most typical or atypical data within a group. The present research work builds on previous research studies that focus on efficient segmentation techniques based on clustering algorithms. As a first study in (Cortina-Januchs, 2011) a solution to the problem of detecting pore spaces in computed tomography (CT) images with the help of image processing is shown, extracting the entities from the images, in addition to using three methodologies for the detection of porous spaces (segmentation), which were (K-Medias algorithm, FCM and SOM neural network). Later he focused on the investigation of (Ojeda-Magaña BQ-D.-B., 2014) in which an alternative method is proposed to detect pore spaces, image processing is also used, data grouping with the help of the PFCM algorithm which segments the images into two groups or regions, one for pore spaces and the other for solid regions. Subsequently, each of these regions is divided into two groups, which are identified as typical and atypical subregions, resulting in four subregions (two typical and two atypical) which constitute the original image, for this approach there is a drawback which is the subjective tuning of the threshold $\alpha$ and the sensitivity of the results to this parameter. To obtain quantitative results of the results of the proposed method, the value of the non-uniformity measure (NU) was used, which is of great help to represent an estimate of the quality of the subsegmentation results, through the variance of the pores with respect to the variance of the whole image. Through the analysis of several applications, it was found that one of the main drawbacks of the segmentation method arises from the fact that two types of atypical pixels of each group were mixed for the representation of the typical object.
This area of opportunity was improved in (B. Ojeda-Magaña, 2018) in which they already differentiate between the two types of atypical pixels, calling the new technique improvement of sub-segmentation, in which the objects of interest are identified better in precision and quantity in addition to improving the ease of selection of the threshold parameter and the robustness against its changes.

\section{Solution model}

This article focuses on the detection of porous spaces in 2D images that have been acquired by means of X-rays or computed tomography. The proposed methodology focuses on digital image processing and data grouping techniques. The images used for pore detection were captured by means of an EVS MS-MicroCT scanner. The images that are generated by the scanner already have the application of a Gaussian filter (retouch filter) which focuses on reducing the noise of the images, but even so there is a problem with the ground images they still contain a bit of Noise and contrast between solid soil and pores is very limited. The first stage focuses on receiving the images of soils with low contrast, the second stage focuses on performing a preprocessing through an image enhancement technique using the adaptive nonlinear transform which improves the contrast between pore spaces and ground. The third stage consists of image segmentation, it focuses on the PFCM hybrid clustering algorithm to find the typical or atypical data within the clusters by means of a resulting matrix of data (Matrix $\mathrm{T}$ ). The fourth and last stage focuses on sub-segmentation which, once the PFCM algorithm is used, the matrix $\mathrm{T}$ is used that segments the pore and soil space regions (2 regions), using a threshold value these regions are divided into subregions, the atypical data of each region are identified, improving homogeneity by reducing the threshold value until the atypical region becomes more homogeneous, where finally the regions of interest are detected, in Figure 1, the diagram is shown. solution model blocks. 


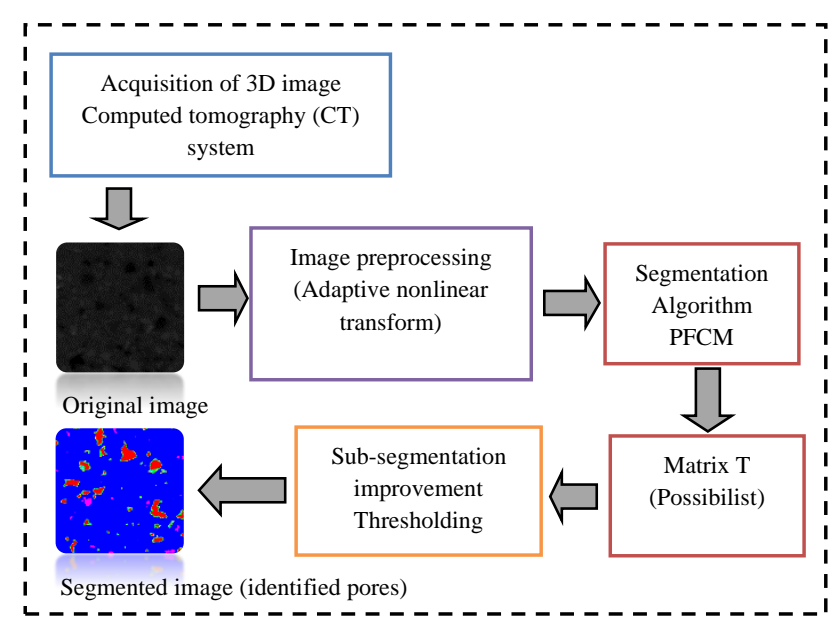

Figure 1 Block diagram for the application of the improvement of subsegmentation in the detection of pores in $\mathrm{CT}$ images

\section{Preprocessing (Contrast Enhancement)}

CT soil images contain noise due to the nature of the acquisition process, it is usually characterized by the small differences in the gray intensity values of the pixels (low contrast) that exist between the pores and soil space, it is possible observe it visually, Figure 2.

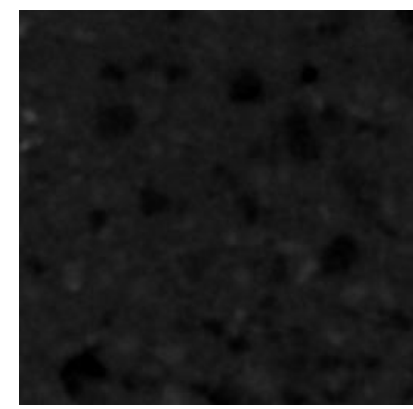

a)

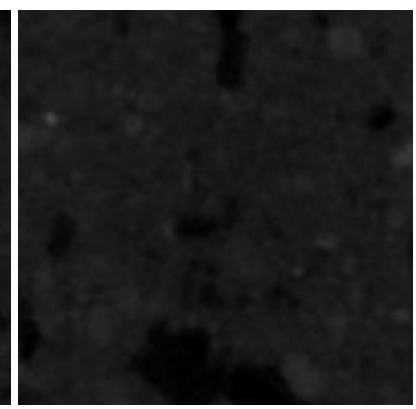

b)
Figure 2 Low contrast 2D images acquired by CT computed tomography, a) image 112 , b) image 343 , with a resolution of $256 \times 256$ pixels

To improve the contrast, a pre-processing stage is pertinent, which reduces the noise and simultaneously increases the values of the pixels that represent the objects of interest. For this process, the adaptive nonlinear transformation is implemented so that the gray values of the pixels are modified and these are closer to the extreme values. The advantage of this technique is that it is able to emphasize the important characteristics of the image while also reducing noise.
This technique has been used in different applications, for example, for the improvement of mammography images (Andrew F. Laine, 1994), the improvement of natural images (Jevtic, 2009) and in ROI images of mammography (Vega-Corona, 2003) in which they use this technique to attenuate the gray levels of the pixels with a small amplitude value. To perform the contrast enhancement, which allows the contrast and threshold to be controlled adaptively, the expression defined in equation (1) is proposed.

$$
\begin{gathered}
I_{T}(x, y)=T(I(x, y))= \\
A[\operatorname{sigm}(k(I(x, y-\beta))-\operatorname{sigm}(-k(I(x, y)+\beta))]
\end{gathered}
$$

Where $\mathrm{I}(\mathrm{x}, \mathrm{y})$ is the gray level of a pixel of the input image $\mathrm{I}_{\text {, }} \mathrm{I}_{\mathrm{T}}$ is the image resulting from the transformation, $\mathrm{T}$ (.) represents the transformation function that acts on $\mathrm{I}(\mathrm{x}, \mathrm{y})$. A is defined as:

$$
\begin{gathered}
A=\frac{1}{\operatorname{sigm}(k(1-\beta)-\operatorname{sigm}(-k(1+\beta))} \\
0<\beta<1
\end{gathered}
$$

and the sigmoidal function is defined with the following equation:

$$
\operatorname{sigm}(x)=\frac{1}{1+e^{-x}}
$$

Where $\beta \in \mathrm{R}$ and $\mathrm{k} \in \mathrm{Z}$ which control the threshold and contrast control in the image respectively. For an input image I, with a maximum gray level L_max, it is necessary to map the image in the range of $\left[-\mathrm{L}_{\max }, \mathrm{L}_{\max }\right]$ to an image in the range of $[-1,1]$, therefore the value of $\mathrm{L}_{\max }$ can be used as a normalization factor. According to the above, the non-linear transformation function equation (1) is represented in graphic 1. 


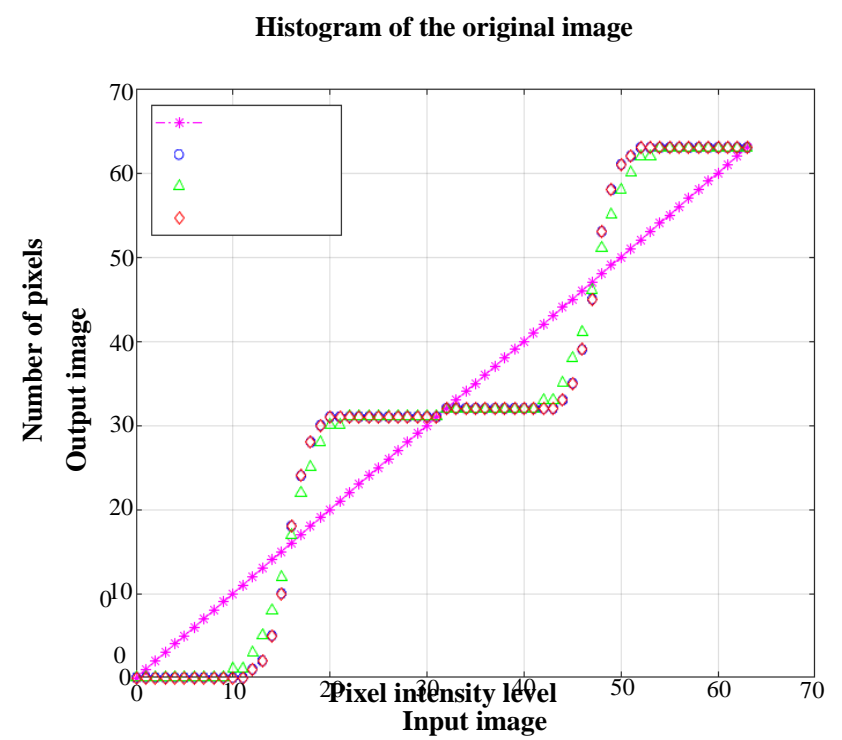

Graphic 1 Nonlinear adaptive transformation function $\mathrm{I}_{\mathrm{T}}$ for contrast enhancement of CT images. This technique was applied in a) image 112, and b) image 343, with parameters $\beta=0.5, \mathrm{k}=10,20,30$.

It is important to mention that values of $\beta$ and $\mathrm{k}$ can be used regardless of the dynamic range of the image, as long as the following restriction is met:

$$
\beta k>2
$$

It is important to mention that values of $\beta$ and $\mathrm{k}$ can be used regardless of the dynamic range of the image, as long as the following restriction is met.

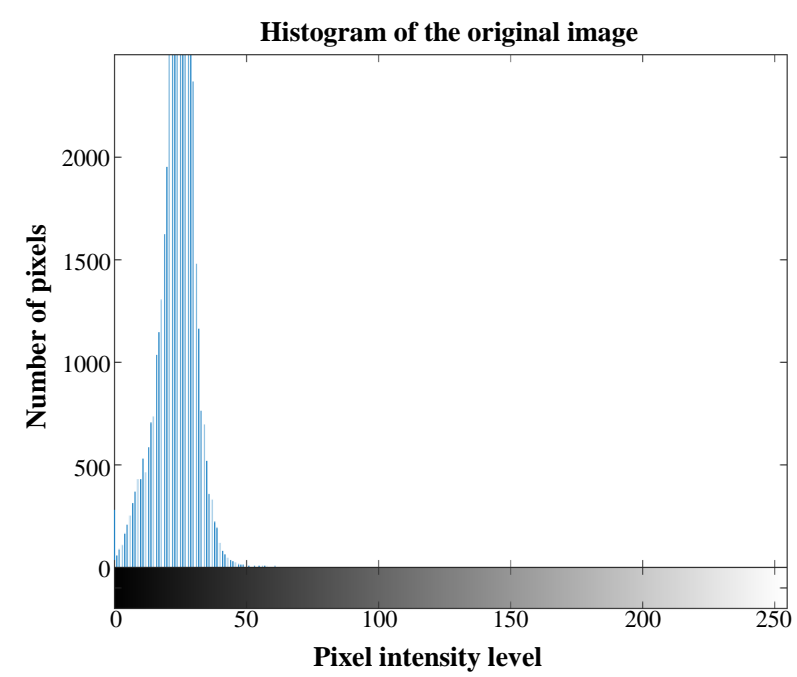

Graphic 2 Histogram where the intensity levels of the pixels of the original image are observed a) Image 112, it is observed that there are pixels with different levels of gray intensity

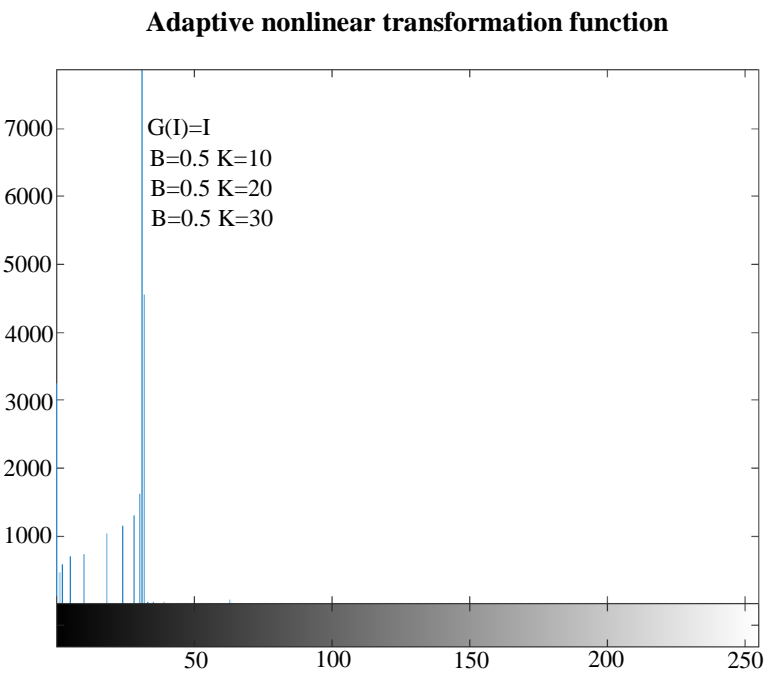

Graphic 3 Histogram where the intensity levels of the pixels of the transformed image a) image 112 are observed, it is observed that the pixels have been grouped and emphasized the important characteristics, the values used are $\beta=0.5, \mathrm{k}=30$

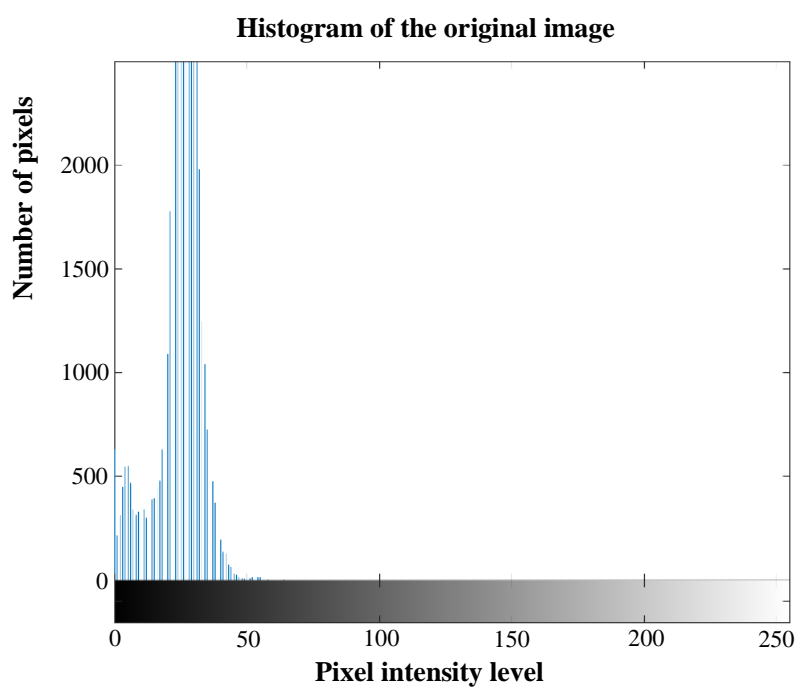

Graphic 4 Histogram where the intensity levels of the pixels of the original image are observed a) Image 343, it is observed that there are pixels with different levels of gray intensity 


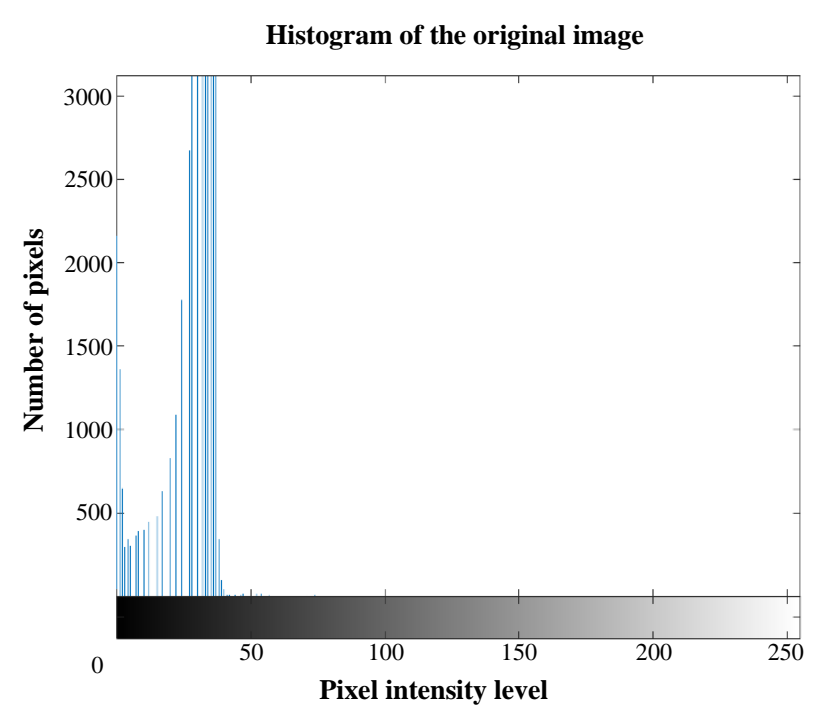

Graphic 5 Histogram where the intensity levels of the pixels of the transformed image a) image 343 are observed, it is observed that the pixels have been grouped and emphasized the important characteristics, the values used are $\beta=0.5, \mathrm{k}=0$

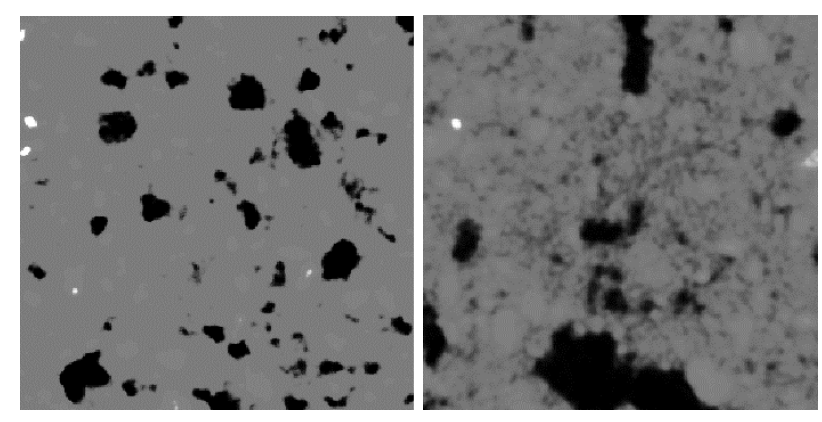

Figure 3 Results of the application of image enhancement, a) image $112(\beta=0.5, \mathrm{k}=30), \mathrm{b})$ image $343(\beta=0.5, \mathrm{k}=$ 10)

\section{Image segmentation using the PFCM algorithm}

The clustering algorithm that has been proposed as a solution to the problems of the FCM and PCM algorithms is the PFCM hybrid algorithm, which has been demonstrated in different experiments by means of a standard data set that is a robust algorithm and that the results are satisfactory. The PFCM algorithm has four adjustment parameters which are: $\mathrm{a}, \mathrm{b}, \eta$ and $\mathrm{m}$. The values of $a$ and $b$ represent the relative importance of membership and similarity in the calculation of prototypes. Therefore, if $a>b$ the prototypes are more strongly influenced by the membership values, otherwise if $b>a$ the typicality values have more influence and it is expected that the prototypes will be less affected by noise. In (Ojeda-Magaña B. Q.-D., 2014) they propose a value of a smaller than $b$ to produce $a$ better identification of the pores.
As for the parameter $\mathrm{m}$ it has the same function as the FCM algorithm, and the parameter $\eta$ has the same function as the PCM algorithm. Current literature recommends that these values be small and less than or equal to two. It is important to mention that when the images are segmented, only two objects will be shown, which are the pore space and the floor space.

\section{Improved sub-segmentation applied to soil images for the detection of pore spaces}

\section{Sub-segmentation}

The method of the new sub-segmentation is based on the concept of typicality which focuses on finding the most typical or atypical data within a group of data and the PFCM hybrid data grouping and partitioning algorithm. This algorithm has been perfected through different research works that have been carried out, in (Ojeda Magaña \& Quintanilla Domínguez, 2009) they make an analogy between the grouping algorithms and the prototype theory that was proposed by (Rosch, 1975 ). The analogy focuses on the degrees of membership of fuzzy sets which can be interpreted as a relative typicality or in other words probabilistic restriction, this means that it results from an external similarity, since the degrees of membership of an object are limited. to unity, in this case 1 . On the contrary, the absolute similarity depends only on the elements of the particular group, due to this the typical or outlier data can be more easily identified within the two target regions (soil and pore). For the subsegmentation of soils, the parameter $\alpha$ is used, which belongs to the threshold that is within the established interval $[0,1]$, through this interval it is possible to divide a region according to the similarity value of each object. The selection of the parameter $\alpha$ must be carefully selected because it establishes the limit between the typical and atypical pixels, therefore, it requires a value close to zero for the atypical pixels and close to one for the typical values, this can be seen more clearly in Figure 4. 


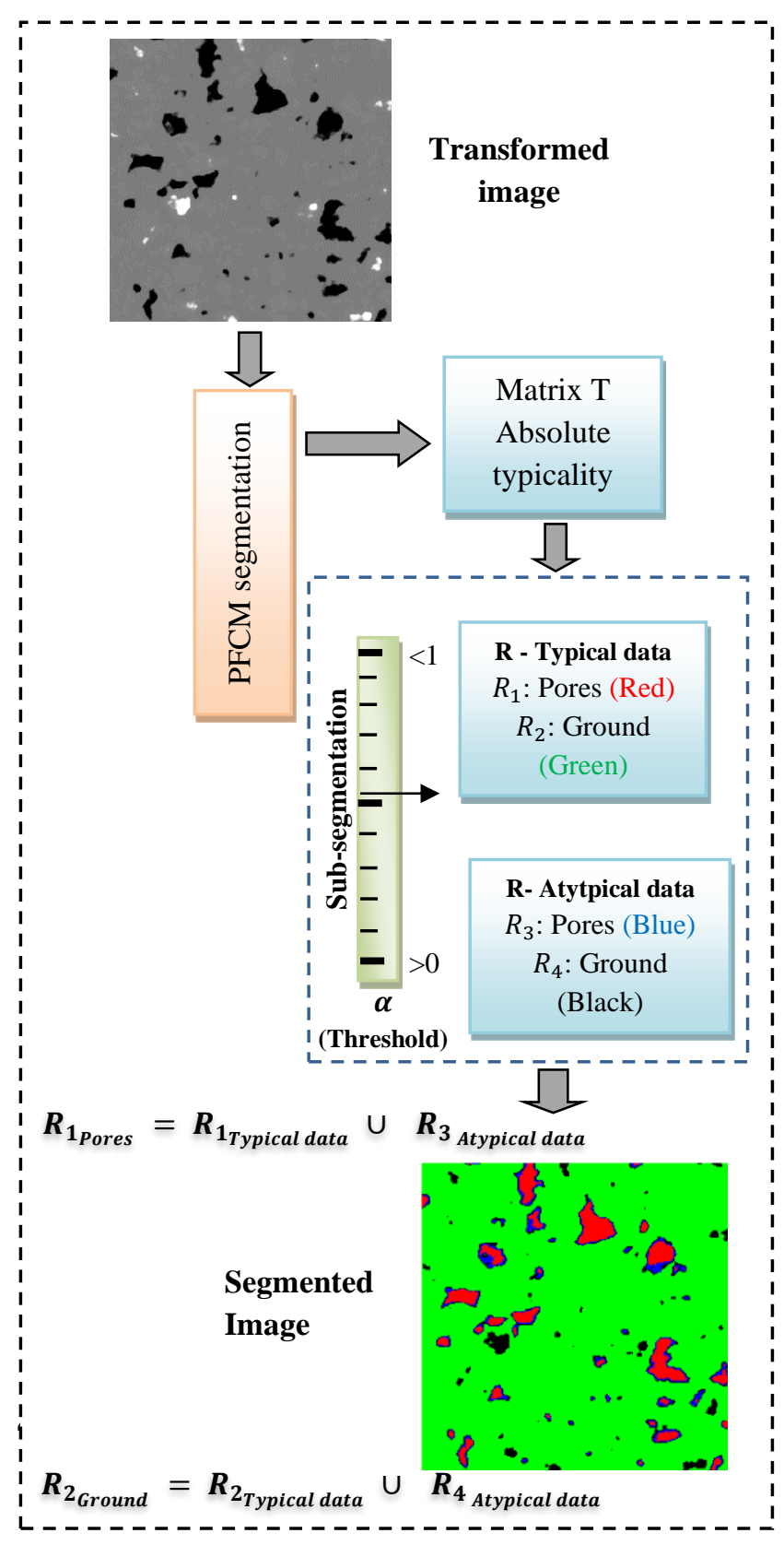

Figure 4 Sub-segmentation process applied to a CT image, image 120 with contrast enhancement parameters $(\beta=0.5, \mathrm{k}=30)$

\section{Improved sub-segmentation}

The sub-segmentation method is a robust method for the detection of pore spaces in CT images, however, through various applications in different fields, the need has been found to not only identify atypical data but also to differentiate between the various atypical data in both groups, this due to the lack of homogeneity despite the fact that there are few pixels in that region. In (B. Ojeda-Magaña, 2018) they mention that to reduce the inhomogeneity of the pixels, the solution is to reduce the threshold value until the atypical region becomes more homogeneous.
They also mention that in some of the applications such as the detection of microcalcifications in mammograms, the regions appear as atypical objects that include values of gray levels close to white. Based on this need, the sub-segmentation process was improved and it was divided into two regions, the atypical pixels of the sub-regions, so this helps in the better detection of the objects of interest. The steps that describe the method of the improved sub-segmentation proposal are as follows:

Given a data set $\mathrm{Z}=\mathrm{Z}_{1}, \mathrm{z}_{2}, \ldots \mathrm{z}_{\mathrm{N}}$ corresponding to the $\mathrm{N}$ pixels in the image, select the number $\mathrm{c}(1<\mathrm{c}<\mathrm{N})$ of clusters or regions $\mathrm{S}_{\mathrm{i}}$, $i=1,2, \ldots, c$, the weight of the exponents $m>1$, $\eta>1$, and the value of the parameters $a>0$ and b> 0 .

\section{Step 1. Run PFCM-AO-V to get:}

(a) The membership matrix $U=\left[\mu_{i k}\right]$

(b) The typicality matrix $\left.\mathrm{T}=\left[\mu_{i k}\right]\right]$

(c) Prototypes $\mathrm{V}=\left[v_{i}\right]$

Step 2. Label each pixel $\mathrm{z} k, \mathrm{~K}=1, \ldots, \mathrm{N}$ according to the fuzzy region (FR), with the maximum membership value in $U=\left[\mu \_i k\right]$, for each $S_{-}(\mathrm{i}(\mathrm{FR})), \mathrm{i}, \ldots, \mathrm{c}$, as shown in equation 4 , so that each pixel z_k can only belong to only one region $S_{-}$(i (FR)). Note: these regions will not be used later after this step, although they are of great importance to the segmentation process.

$$
S_{i(F R)}=\max _{i}\left[\mu_{i k}\right], \quad i=1, \ldots, c
$$

Step 3. Label each pixel $z_{k}, K=1, \ldots, N$ according to the possible region (PR) with the maximum typicality valued in $\mathrm{T}=\left[\mu_{i k}\right]$, for each $S_{i(P R)}, i, \ldots, c$, as shown in equation 5 . Such that each pixel $z_{k}$, can only belong to one region $S_{i(P R)}$ for possible regions.

$$
S_{i(P R)}=\max _{i}\left[t_{i k}\right], \quad i=1, \ldots, c
$$

Step 4. Obtain the maximum typicality value for each point (pixel) of the previous one $S_{i(P R)}$ matrix and put it in the vector $T_{\text {max }}$ :

$$
T_{\max }=\max _{i}\left[t_{i k}\right], \quad i=1, \ldots, c
$$


Step 5. Label the pixels $z_{k}$ according to the typicality value of the subvector $T_{1}$ to get the following subregions:

$$
S_{i-\text { typical }}=\left\{\begin{array}{l}
\text { i, if } t_{i k}=T_{1} i=1, \ldots, c \\
0, \text { otherwise }
\end{array}\right.
$$

Step 6. Map the outlier pixels, according to their typicality value, using only the subvector $T_{2}$ equation 8 . Then map the typicality of this sub vector to gray values of the corresponding pixels. Then a new sub-vector is generated $T_{2 \text { (new) }}$.

Step 7. Differentiate from prototypes $v_{i}$ from each region $S_{i(P R A)}$ of the atypical pixels of $T_{2(\text { new) }}$. Separate the pixels and save the corresponding labels in two sub-vectors $S_{i-}$ atypicalRegion $_{c+i}, S_{i-}$ atypicalRegion $_{2 c+i}$ where the labels of the first sub-vector are selected according to:

$S_{i-}$ atypical $_{c+i}=\left\{\begin{array}{l}i, \text { if }=T_{2(\text { new })} \geq v_{i} \\ 0, \text { otherwise }\end{array}\right.$

Which will contain the darkest outlier pixels in region $i$. On the other hand, the elements of the second sub-vector are selected according to:

$S_{i-}$ atypical $_{2 c+i}=\left\{\begin{array}{l}i, \text { if }=T_{2(\text { new })}<v_{i} \\ 0, \text { otherwise }\end{array}\right.$

Which contain the brightest outlier pixels in region i. So each region $P R A_{\text {new }}$ is defined by:

$S_{i(\text { PRAnew })}=S_{i-}$ typical $_{i} \cup S_{i-}$ atypicalRegion $_{(c+i)}$

$\cup S_{i-}$ atypicalRegion $_{(2 c+i)}$

Step 8. Select the sub-vector $\mathrm{T}_{-} 1$ or the sub-regions $\left(S_{i-}\right.$ atypicalRegion $_{(c+i)}$,

$\left(S_{i-}\right.$ atypicalRegion $\left._{(2 c+i)}\right)$ of interest for the corresponding analysis.
To improve the sub-segmentation, the matrix $\mathrm{T}_{(2}$ new) is taken but now with the gray value of the corresponding pixels, so that an order relationship is established with the prototype of each region, from that it is It is possible to establish an order with the prototypes of each region and it is possible to divide the pixels into two groups, the darkest atypical pixels and the brightest atypical pixels.

\section{Results}

The use of digital processing techniques such as contrast enhancement through adaptive nonlinear transform to prepare gray intensity levels as well as segmentation through the PFCM hybrid clustering algorithm and subsegmentation enhancement help To detect the porosity structure in soil images acquired by $\mathrm{CT}$ and for this, all the pores of all the images of the sequence that have been taken to each soil sample are required. Normally the soil structure is represented by the pixels with the lightest colors, while the pores are represented by the darkest pixels. In Figure 10 two original images of the ground with low contrast are shown and later the processed image to which the contrast enhancement has already been applied where it is observed that the contrast has been significantly improved, later images are also attached to which they are The process of conventional sub-segmentation has been applied to them. The parameters selected for the PFCM algorithm were: $a=1, b=2, m=2$ and $\eta=2$ while the threshold that was selected is relatively low $\alpha=0.08$ for the conventional subsegmentation method, this is due to that the object of interest or the atypical subregion to be identified are the pores or pore spaces. The parameters that were chosen for the improvement of the sub-segmentation were $(\mathrm{c}=$ $2, \mathrm{a}=1, \mathrm{~b}=2, \mathrm{~m}=2$ and $\eta=2$ ) and the threshold parameter $\alpha=0.1$ is chosen again a close value zero to detect pore outliers. For the conventional sub-segmentation method, each image is segmented into two regions (pore and soil), subsequently it is sub-segmented into four subregions, two typical regions and two atypical regions, the results are shown in Figure 5. 


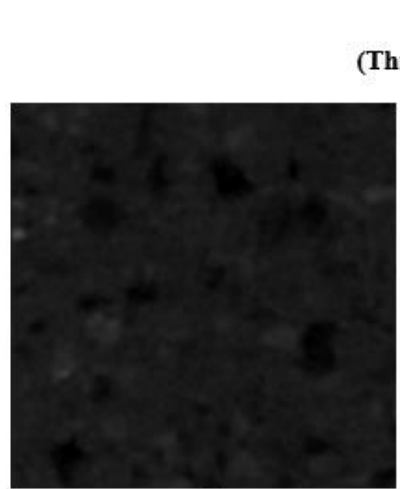

a)

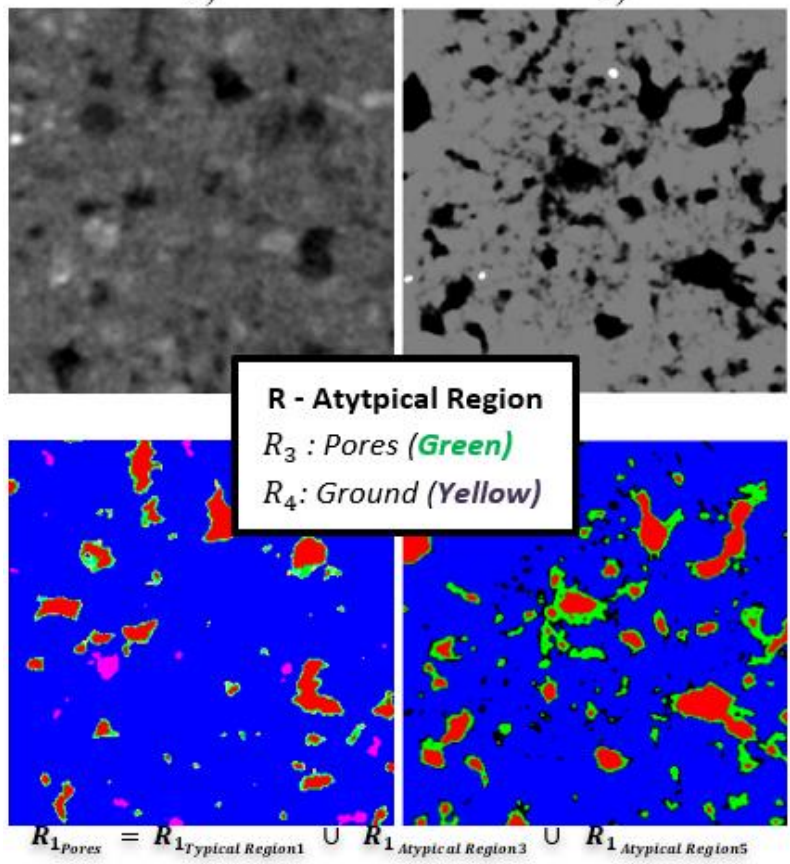

Figure 5 Contrast enhancement process applied to two CT images, a) image 116 with the parameters $(\beta=0.5, \mathrm{k}=10)$, b) img 148 with the parameters $(\beta=0.5, \mathrm{k}=30)$, for the process of sub-segmentation the parameters $\mathrm{a}=1, \mathrm{~b}=2$, $\mathrm{m}=2$ and $\eta=2$ were used, with a threshold $\alpha=0.08$

For the sub-segmentation improvement method, based on the two groups of pixels (pore and soil), in each of them there are three subregions (one typical region and two atypical regions), resulting in six sub-regions that provide more information of interest about the study that is being carried out and provide even more information than the sub-segmentation method. Figure 6 shows the methodology of this process.

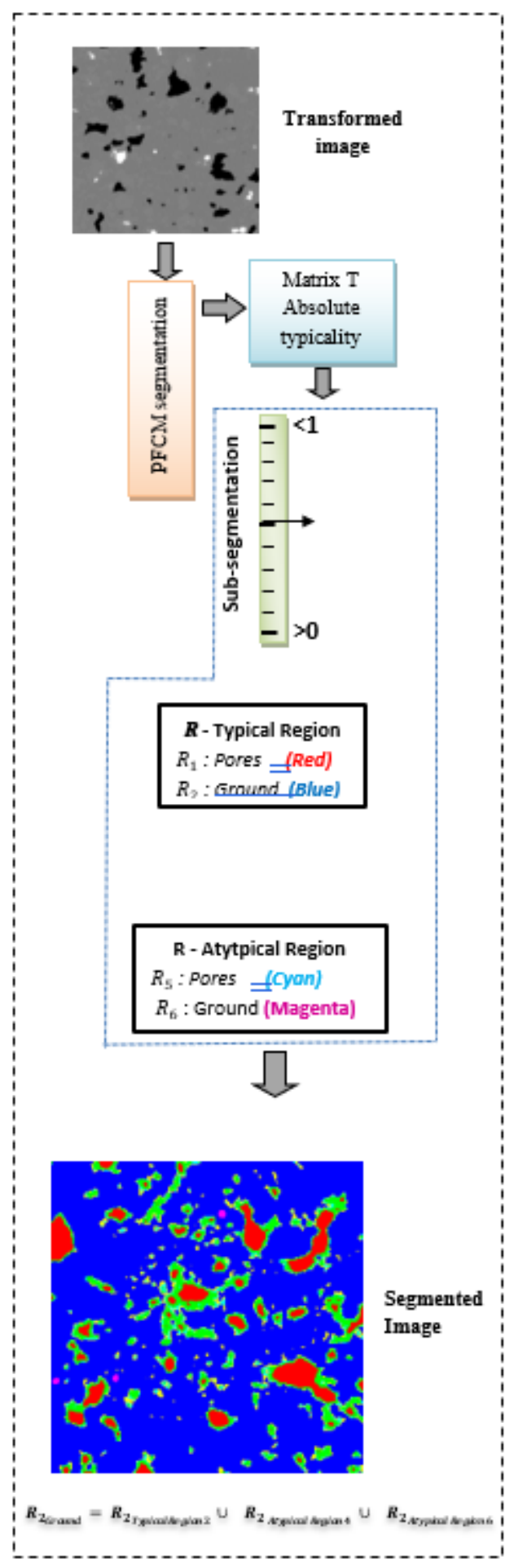

Figure 6 Sub-segmentation improvement process applied to a CT image, image 148 with contrast enhancement parameters $(\beta=0.5, \mathrm{k}=30$. In this process, the six subregions into which the image is divided are clearly observed and the contribution of more information of interest

A result of the contrast enhancement and the enhancement of the subsegmentation of the CT 148 image is also shown, in Figure 7. 


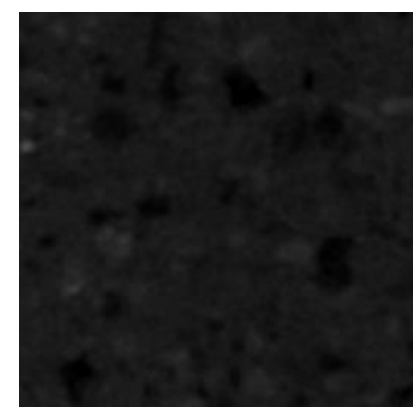

a)
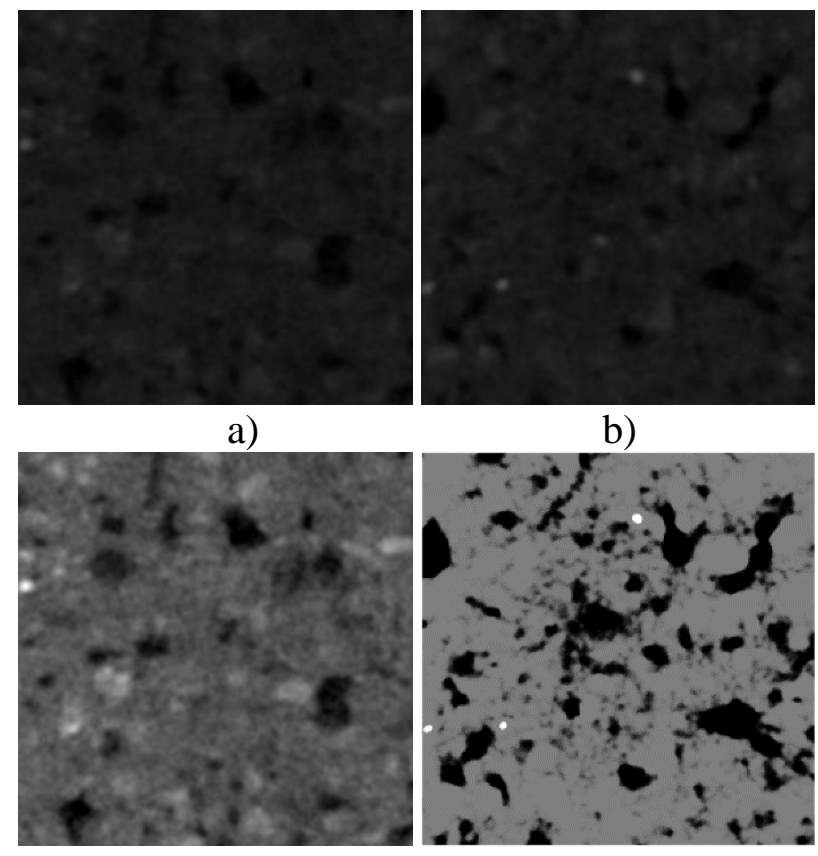

b)
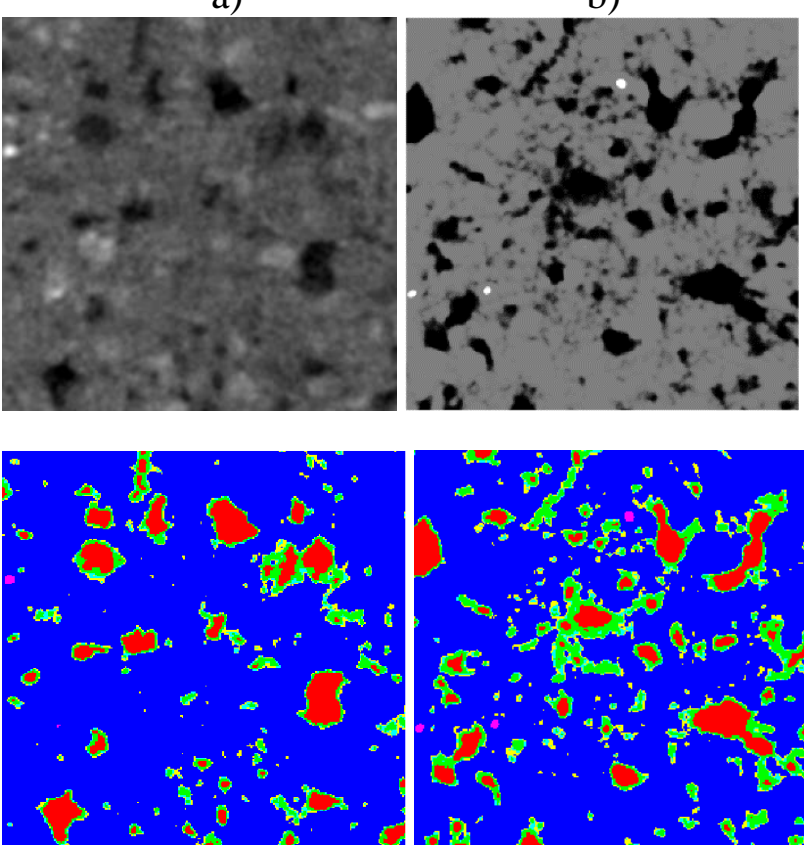

Figure 7 Contrast enhancement process applied to two CT images, a) image 116 with the parameters $(\beta=0.5, \mathrm{k}=10)$, b) image 148 with the parameters $(\beta=0.5, \mathrm{k}=30)$, for the process of the improvement of sub-segmentation, the parameters $\mathrm{a}=1, \mathrm{~b}=2, \mathrm{~m}=2$ and $\eta=2$ were used, with a threshold $\alpha=0.1$

Preprocessing for contrast enhancement, segmentation by means of the PFCM clustering algorithm, as well as conventional subsegmentation and sub-segmentation enhancement have been applied to 256 images (image 100 - image 356), keeping the parameters contrast enhancement and PFCM algorithm used in this results section. For conventional subsegmentation a threshold of 0.08 and in the case of sub-segmentation improvement a threshold of 0.2 .

\section{Conclusions}

With the help of digital signal processing and data grouping algorithms it is possible to segment images to find regions of interest and by means of a threshold which is applied to the conventional sub-segmentation technique and the improvement of sub-segmentation.
It is possible to find subregions of interest that focus on the typicality and nontypicality of the data, which promotes reaching a more concise and precise result than a particular segmentation. An advantage of subsegmentation techniques is that the threshold can be adjusted in a range of $[0,1]$ so that objects can be adjusted to these threshold levels, while the other methods search through a number. discrete groups in order to approximate objects of interest. The tests shown in the present research work show promising results and verify that the methodology shown here is quite robust for the segmentation of soil images, for the moment the inspection analysis of the results of the segmentations carried out here have been evaluated by the visual inspection method, for which reason it is subsequently intended to implement a quantitative analysis method to verify the homogeneity of porosity of the segmented soil images, as well as the development of synthetic images to perform the quantitative analysis, this because there is no there is an ideal comparison of a real image by the nature of the image acquisition.

\section{Acknowledgments}

The authors thank the Polytechnic University of Juventino Rosas for financing the publication of the article, the University of Guadalajara, the Polytechnic University of Madrid, the National Council of Science and Technology (CONACyT) as well as the researchers who contributed in the review and writing of the manuscript of the article.

\section{References}

Andrew F. Laine, S. S. (1994). Mammographic Feature Enhancement by Multiscale Analysis. IEEE TRANSACTIONS ON MEDICAL IMAGING, 725-739.

B. Ojeda-Magaña, J. Q.-D. (2018). Improvement of the Image Sub-Segmentation for Identification and Diferentiation of Atypical Regions. World Scientific, 26.

Barni, M. C. (1996). A possibilistic approach to clustering. IEEE Trans. Fuzzy, 393-396.

Bezdek, J. C. (1981). Pattern Recognition with Fuzzy Objective Function. Plenum Press. 
C.J. Bronick, R. L. (2005). Soil structure and management: a review. Geoderma, 3-22.

Cortina-Januchs, M. Q.-D.-C. (2011). Detection of pore space in CT soil images using artificial neural networks. Biogeosciences, 279-288.

Crawford, J. (2010). Can complex be simple? Biogeosciences, 1-2.

De Bartolo, S. O. (2011). Modeling soil system: complexity under your feet. Biogeosciences, 3139-3142.

Dunn, J. C. (1973). A Fuzzy relative of isodata process and its use in detecting. J. Cybernetics, $32-57$.

Hamamoto, M. P. (2011). Extreme compaction effects on gas transport parameters and estimated. J. Geotech. Geoenviron. Eng., 653663.

Jevtic, A. Q.-D.-J. (2009). Edge detection using ant colony search algorithm and multiscale contrast enhancement. In The 2009 IEEE International Conference on Systems,Man, and Cybernetics, 2193-2198.

Krishnapuram, \& R., Keller, J.M. (1993). A possibilistic approach to clustering. IEEE Trans.Fuzzy Syst., 98-110.

M. G. Cortina-Januchs, J. Q.-D.-C. (2011). Detection of pore space in CT soil images using artificial neural networks. Biogeosciences, 279288.

MacQueen, J. B. (1967). Some Methods for classification and Analysis of Multivariate Observations. Proceedings of 5-th Berkeley Symposium on Mathematical Statistics and Probability, 281-297.

Magaña, O. (499-503). Images subsegmentation with the PFCM clustering algorithm INDIN 2009. 7th IEEE International Conference, 2009.

Moreiro, J. (2002). Aplicaciones al análisis automático del contenido provenientes de la teoria matemática de la información. Anales de Documentación, 273-286.
Ojeda Magaña, B., \& Quintanilla Domínguez , J. (2009). Images sub-segmentation with the PFCM clustering algorithm INDIN 2009. 7th IEEE International Conference, 499-503.

Ojeda-Magaña, B. Q.-D. (2014). Identification of pore spaces in 3D CT soil images using PFCM partitional clustering. Geoderma, 90-101.

Ojeda-Magaña, B. Q.-D.-B. (2014). Identification of pore spaces in 3D CT soil images using PFCM partitional clustering. Geoderma, , 90-101.

Pal, N. P. (2005). A possibilistic fuzzy c-means clustering algorithm. IEEE Trans. Fuzzy Syst., 517-530.

Perret, J. P. (2000). A two-domain approach using CAT scanning to model solute transport in soil. . J. Environ. Qual., 995-1010.

Rosch, E. M. (1975). Family resemblance: studies in the internal structure of categories. Cogn. Psychol, 573-605.

Santos Peñas, M., \& Farias Castro, G. (2010). Laboratorios virtuales de procesamiento de señales. Revista Iberoamericana de Automática e Infórmatica Industrial, 91-100.

Vega-Corona, A. Á.-V. (2003). Feature vectors generation for detection of microcalcifications in digitized mammography using neural networks. In Artificial Neural Nets Problem Solving Methods, vol. 2687 of Lecture Notes in Computer Science, 045-1045.

Vogel, H. J. (1996). Topological characterization of pore space in soil-sample preparation and digital imageprocessing. Geoderma, 23-38.

Wakene C. Negassa, A. K. (2015). Properties of Soil Pore Space Regulate Pathways of Plant Residue Decomposition and Community Structure of Associated Bacteria. PLOS ONE, 122

Zadeh, L. (1965). Fuzzy sets. Inf. Contro, 338353.

ARREGUIN-JUÁREZ, Miguel, QUINTANILLADOMINGUEZ, Joel, OJEDA-MAGAÑA, Benjamín and TARQUIS-ALFONSO, Ana María. Application of subsegmentation enhancement in pore detection in soil CT images. Journal of Technology and Innovation. 2021 